\title{
ACOMPANHAMENTO PSICOLÓGICO TARDIO EM PACIENTES SUBMETIDOS À CIRURGIA BARIÁTRICA
}

\author{
Late psychological follow-up on patients submitted to bariatric surgery
}

Simone Dallegrave MARCHESINI

Trabalho realizado na Clínica Marchesini, Curitiba, PR, Brasil

DESCRITORES - Obesidade mórbida; Cirurgia bariátrica. Avaliação. Recidiva.

\section{Correspondência:}

Simone Dallegrave Marchesini, e-mail: simonedallmarc@yahoo.com.br

Fonte de financiamento: não há Conflito de interesses: não há

Recebido para publicação: 03/11/2009 Aceito para publicação: 19/01/2010

HEADINGS - Obesity, morbid. Bariatric surgery. Evaluation. Recurrence.
RESUMO - Racional - O ser humano não se constitui só; existe sempre em relação aos seus pares e ao meio-ambiente. Toda mudança corresponde a uma reação social e a uma resposta emitida em forma de comportamento. Objetivo - Avaliar as condições pósoperatórias mediatas e tardias de pacientes bariátricos em contexto global e compreender sua psicologia. Método - Foram enviados questionários contendo 26 quesitos abrangendo motivação, aderência ao tratamento, mudanças de comportamento, alterações de humor, alcance das expectativas entre outros. Resultados - A maioria dos pacientes bariátricos sente-se preparada para a cirurgia bariátrica na primeira entrevista psicológica, mas não conhece o método ao qual será submetida. Grande parte não tem tratamento para o aspecto psicológico/psiquiátrico que acompanha a doença e não muda o estilo de vida após a operação. A adesão às recomendações nutricionais é parcial e ocorrem alterações emocionais ligadas às deficiências dessa ordem. Em comparação com a satisfação em perder peso a recuperação de peso é considerada pequena. Quase todos os entrevistados fariam a operação novamente e metade vê necessidade de grupos de apoio. Observou-se que os pacientes desejam a operação, mas não percebem a necessidade de maior preparação para o procedimento e não conseguem mudar padrões de comportamento necessários para o sucesso em longo prazo, pois abandonam o tratamento. Conclusão - Pode-se inferir que as motivações que levam os indivíduos a procurar a cirurgia bariátrica são calcadas nos retornos sociais, nos padrões estéticos e no desejo psicológico de mudança de vida. Uma vez concretizada a mudança de vida, a motivação se acaba, mas não garante a satisfação existencial. Também é possível inferir que o preparo para o atendimento de cônjuges e familiares reclama um espaço na formação de profissionais da área do tratamento cirúrgico da obesidade, para que a prevenção às recaídas possa ser foco terapêutico.

ABSTRACT - Background - The human being is not alone; he is always compared to their peers and the environment. Every change corresponds to a social reaction and a response issued in the form of behavior. Aim - To evaluate some of the postoperative psychological conditions and life style in a short and in a long term follow-up. Method - A 26 item evaluation questionnaire was sent to post bariatric patients concerning to motivation and compliance to the treatment, behavioral changes, humor alterations, reaching of expectancies among others. Results The majority of this group of patients felt prepared for surgery during the first office interview, but didn't know the method of treatment that they were about to be submitted to. The majority also had not been treated for their psychological and/or psychiatric conditions and didn't change their life style after the operation. Compliance to nutrition orientation was partial and there were evident emotional manifestations. The weight regain complaint was small compared with the satisfaction of the weight loss. Most of these patients would be submitted again to the operation, and half of them stated the need for support groups. Patients wanted the operation, but didn't realize the need of better preparation and didn't change the behavioral patterns necessary to reach the success in a long term follow-up. They usually abandon the postoperative treatment. Conclusion - It can be inferred that the motivations that lead individuals to seek bariatric surgery are predicated on social returns, in the aesthetic standards and the psychological desire for life change. Once implemented the change of life, motivation is over, but does not guarantee the existential satisfaction. It is also possible to infer that the preparation for the care of spouses and family claims a space in training professionals in the area of surgical treatment of obesity, for the prevention of relapses can be therapeutic focus. 
INTRODUÇÃO

A comunicação entre profissionais de saúde e pacientes sempre teve seus obstáculos, mas existem características específicas do grupo bariátrico. A expectativa em relação aos resultados sobre a auto-estima e motivação ainda prevalece na maioria dos pacientes, enquanto ofococentral daequipemultidisciplinar permanece na resolução das doenças associadas à doença obesidade. Entre os operados se destacam aqueles submetidos aos procedimentos cirúrgicos há mais tempo. A conduta humana frente à realização de um ideal ergue diante da equipe multidisciplinar barreiras camufladas pela impulsividade e pela compulsão iniciais, mostradas nas primeiras entrevistas. Em nome da realização da operação presenciam-se múltiplas promessas pré-operatórias que apontam para o desejo prestes a ser realizado. No entanto, fica evidente no decorrer dos anos, que a motivação só permanece na proporção em que o objetivo não é alcançado. Uma vez eliminada a obesidade não há por que se empenhar contra ela. Os candidatos cirúrgicos, quando passam à condição de operados bariátricos, esquecem dos propósitos do tratamento.

O ser humano não se constitui só; existe sempre em relação aos seus pares e ao meio-ambiente. Toda mudança corresponde a uma reação social e a uma resposta emitida em forma de comportamento. Desta feita, a pessoa que perde peso, aumenta seu senso de valor próprio, se traja com maior propriedade, sente-se mais adequada, é percebida como objeto de desejo. Tende a pensar o mundo, a sentir a vida e a emitir comportamentos diferenciados. As variações do comportamento, para mais ou para menos, apontam para possíveis distúrbios que devem ser analisadas com detalhes.

A restrição alimentar determinada pelo volume gástrico ou a purgação via vômito e/ou diarréia buscam um sentido existencial para se sustentarem de modo producente. Sem significado na vida de cada operado existirão casos frustrados, pela perspectiva psíquica. $O$ incentivo externo tem ação de mola propulsora, mas a persistência na aderência depende da recontratação periódica consigo próprio. A figura do terapeuta, seja externa ou já internalizada, é veículo para que mudanças processadas sejam mantidas ao longo do tempo.

O objetivo desta pesquisa foi avaliar as condições pós-operatórias mediatas e tardias de pacientes bariátricos em contexto global e compreender melhor sua psicodinâmica.

\section{MÉTODO}

Com referência nas mudanças psicológicas observadas ao longo do trajeto dos pacientes bariátricos, foram elaboradas perguntas para compor um questionário, na tentativa de avaliar a compreensão dos pacientes acerca dos procedimentos aos quais foram submetidos; demarcar mudanças emocionais processadas ao longo da perda de peso; ressaltar transformações na qualidade de vida; destacar alterações na relação com a alimentação ou deslocamento para outra gratificação e, ainda, verificar a adesão ao tratamento de modo integral.

A amostra se delimitou a 46 pacientes de ambos os sexos operados em épocas diferentes. A população foi de pacientes de clínica privada em Curitiba.

Foram enviados questionários via internet com 26 questões, sendo 16 delas do tipo "sim ou não" e 10 questões de múltipla escolha que não se eliminavam entre si, podendo, portanto ser marcadas mais de uma alternativa. As perguntas tiveram como conteúdo geral, "mudanças comportamentais e estados afetivos". Os participantes foram informados que estavam participando de uma pesquisa com finalidade de publicação e, que a veracidade das informações era de extrema importância para a orientação de futuros pacientes. Todos tiveram consentimento informado aprovado (Figura 1).

\section{RESULTADOS}

Dos 90 questionários enviados através de e-mails, muitos retornaram devido a caixas postais cheias e mudanças de endereços eletrônicos. Foram obtidas 46 respostas, muitas com comentários e depoimentos pessoais anexados e solicitação de acesso ao resultado da pesquisa. Por constituir-se de 26 questões que repetem alguns tópicos ao longo de sua trajetória, foi possível detectar contradições em temas de maior subjetividade.

De acordo com os resultados obtidos e considerando a subjetividade da avaliação, aproximadamente $43,5 \%$ dos pacientes não compreenderam que o procedimento conhecido popularmente como redução do estômago inclui a derivação intestinal. Ficou claro que a restrição gástrica ainda é o elemento marcante da operação de by-pass em Y de Roux. Dos entrevistados 89\% sentiam-se preparados para a cirurgia bariátrica no dia da entrevista psicológica, porém $26 \%$ admitiram terem passado pelo "dia do arrependimento" diante de desconforto ou limitação provocada pelo procedimento e $67,4 \%$ admitiram que deveriam ter se preparado psicologicamente melhor, com mais do que uma avaliação psicológica.

No que tange à motivação, a saúde debilitada preencheu a porcentagem de $30,4 \%$ como critério para decisão para a cirurgia bariátrica, mas ainda a mola propulsora dos pacientes é a discriminação social e estética com $54,3 \%$ e o desejo de iniciar uma nova etapa na vida 45,6\%. O baixo índice de separações conjugais ou divórcios levaram à suposição de que, pessoas que rompem as relações afetivas, já tinham conflitos quanto aos relacionamentos e a operação serviu apenas como incentivo para ir ao ato. Dentro da classificação dos Transtornos do Humor a Depressão foi a mais pontuada, com $21,7 \%$ de casos em tratamento pré-operatório. Sem doença mental relatada no pré-cirúrgico foram marcados $45,7 \%$, enquanto 
1) Você se submeteu a 1 ou mais procedimentos bariátricos? ( ) Alterou para melhor assim que a dor passou

( ) somente um

( ) mais de um

2) Você se submeteu a quais dos procedimentos abaixo?

( )Balão intragástico

( )Balão intragástrico e Cirurgia de Fobi-Capella

( )Balão intragástrico e Cirurgia de Scopinaro

( )Balão intragástrico e Duodenal-Switch

( )Cirurgia de Capella (grampos)

( )Cirurgia de Capella e redução do intestino

( ) Duodenal-Switch

( ) Só sei que é a cirurgia do estômago.

( ) Não sei o nome.

( ) Não responderam 3) Volte ao dia em que você foi à entrevista psicológica para ( ) Sim, estou bebendo muito.
avaliação pré-operatória. Você se achava preparado ( ) Sim, estou comprando muito. emocionalmente para fazer sua operação? ( ) Sim, estou comendo e vomitando muito.

( ) $\operatorname{sim}$

( ) não

( ) Sim, estou trocando muito de parceiras (os)

( ) Sim, estou jogando muito

4) Hoje, que a operação já foi feita, você acha que foi mais ( ) Sim, estou praticando exercícios exageradamente.

motivado (a) por quais dos fatores abaixo? ( ) Sim, estou consumindo medicações exageradamente.

( ) Discriminação social e estética

( ) Saúde debilitada

( ) Situação conjugal estremecida

( ) Depressão

( ) Desejo interno de iniciar uma nova etapa na vida

$\begin{array}{ll}\text { ( ) Não queria mais ser gordo } & \text { ( ) Perícia } \\ \text { ( ) Pelos limites físicos que tinha para fazer o que gostava ( ) Retorno da anestesia }\end{array}$ de fazer.

( ) Nada do acima citado.

( ) Nenhuma das acima.

( ) Não responderam

11) Qual você considera a pior fase da operação?

( ) Fase dos exames

( ) Perícia

( ) Quinze dias dos líquidos

5) Você fazia algum tratamento para controlar problemas ( ) O início da mastigação da área emocional?

( ) Para a depressão

( ) Para a ansiedade

( ) Para transtorno do pânico

( ) Para compulsão

( ) Para transtorno bipolar

( ) Para a insônia

( ) Para transtorno explosivo

( ) Para transtorno compulsivo alimentar

( ) Nenhum dos acima

( ) Não responderam

6) Que tipo de tratamer quadro emocional?

( ) Psicoterapia isoladamente

( ) Psicoterapia e medicação

( ) Somente medicação

( ) Nenhum

( ) Não Responderam

7) Você já sabia do fazer a operação?

( ) $\mathrm{Sim}$

( ) Não responderam

( ) Não

8) Após a operação seu estado emocional se alterou?

( ) Sim, mas somente no hospital

( ) Sim, no período da dieta líquida

( ) $\mathrm{Sim}$, nos primeiros 30 dias

( ) Sim, nos primeiros seis

( ) Sim, no primeiro ano
( ) Dosar o alimento no novo estômago

( ) Pensar em plásticas e em novo internamento.

( ) Não responderam

12) Você conseguiu seguir as orientações nutricionais?

( ) Sim, totalmente

() Sim mas somente as orientações dietéticas (comida), não 22) No tomei suplementos.

( ) Sim, mas só quanto aos suplementos, não segui a dieta

( ) Não do tipo:

( ) "Comer e vomitar"

( ) "Mastigar e dispensar o bagaço da comida"

( ) "Perder o prazer de comer"

() "Evitar situações que sejam concentradas no ato de comer: ( ) Sim jantares, almoços, coquetéis." ( ) Não

( ) Nenhuma das alternativas

( ) Não responderam

vassou por todo o procedimento e ( ) Sim que alguns serviços fazem seis meses de preparo pré- ( ) Não operatório, você acha que as consultas psicológicas 26) Nós formamos uma equipe que não trabalha com poderiam ser estendidas para quatro a seis ao invés de grupos de apoio para falar de questões comuns aos ficar em uma única antes da operação?

( ) $\operatorname{Sim}$

( ) Não

15) Existe um grande número de divórcios após a cirurgia bariátrica. Isso aconteceu com você?
16) Você acha que seu parceiro (a) estava preparado (a) para ter ao lado dele (dela) uma pessoa magra com auto-estima melhor?

Não responderam

( ) Não

17) Você acha que sua família estava preparada para conviver com você mais magro (a) e com melhor autoestima, e com todas as consequências disso? ) $\operatorname{Sim}$

( ) Não

19) Mesmo sua auto-estima estando em alta após a cirurgia bariátrica, você pode afirmar que seu desejo sexual está melhor?

Não

20) Você perdeu o peso que esperava perder?

( ) $\mathrm{Sim}$

21) Você mudou seu estilo de vida adicionando hábitos saudáveis do tipo exercícios físicos regulares e com orientação profissional?

( ) $\mathrm{Sim}$

2) Você percebe ou alguém critica você por estar praticando exercícios exageradamente, de modo até Sim

23) Você recuperou peso após a operação?

( ) $\mathrm{Sim}$

) $\mathrm{Sim}$

25) Você acha que existe preconceito em relação ao operado bariátrico?

FIGURA 1 - Questionário enviado via internet para resposta dos pacientes

os demais relataram outras doenças pertinentes aos Transtornos de Ansiedade e Transtornos Alimentares. Na ocasião do preenchimento do questionário $51,2 \%$ estavam sem nenhum tratamento no plano psicológico/psiquiátrico e os que tinham tratamento davam preferência à psicoterapia isoladamente $19,6 \%$, e medicação psiquiátrica isoladamente $13 \%$. Do total de pacientes, $69,6 \%$ admitiram já saberem do diagnóstico emocional antes de passarem pelo procedimento cirúrgico, o que demonstra a resistência em admitir o vínculo entre $o$ ato alimentar e os estados de humor. O deslocamento da compulsão para outro foco superou em metade da amostra (56,5\%). Aproximadamente $11 \%$ relataram substituir a comida por bebida e a mesma porcentagem estar comprando excessivamente; 6,5\% relataram estar comendo e vomitando e a mesma porcentagem relatou estar trocando com frequência de parceiros. Houve melhoria do padrão da vida sexual em $71,7 \%$ dos pacientes que responderam à pesquisa. Nenhum derivou sua impulsividade ou compulsão para a prática de exercícios físicos. A pior fase do tratamento cirúrgico da obesidade foi considerada por $39 \%$ dos pacientes a dieta líquida pós-operatória. Nessa fase de 15 dias a restrição alimentar é radical após hábito de consumo calórico elevado; a falta da mastigação e a redução de nutrientes transformam esse período em ritual de passagem. O retorno anestésico ficou em segundo lugar como pior período do tratamento operatório para a obesidade e marcou $32,6 \%$ dos pacientes. $A$ adesão às orientações nutricionais foi exatamente de $50 \%$ e os demais pacientes se dividiram entre seguir apenas as orientações dietéticas ou apenas tomar a suplementação. Ainda existe na população de operados ou submetidos a tratamentos 
de emagrecimentos variados a idéia de que vitaminas são "engordativas". Aproximadamente $61 \%$ negaram ter desenvolvido algum comportamento alimentar exótico ou distúrbio do comportamento alimentar após a cirurgia bariátrica. Mas dos comportamentos desenvolvidos, o mais apontado foi "comer e vomitar" com 8,7\% de referência. Os pacientes admitiram que $32,6 \%$ dos parceiros não estavam preparados para ter magros ao seu lado e 17,4 se divorciaram após a operação. Alguns pacientes deixaram claro que a idéia da separação estava inclusa no desejo de mudar de vida, não sendo a operação a causa do divórcio, mas facilitadora do ato. Quanto à família, 19,6\% perceberam que ela não manejou bem a transformação do operado bariátrico. Quanto à necessidade de ser aprovado e aceito, 43,5\% admitiu não mais ter a necessidade de compensar com outros adjetivos o fato de ser obeso. Embora seja quesito necessário para a manutenção dos resultados cirúrgicos, 67,3\% admitiram que não mudaram seus hábitos e estilo de vida, mas $93,5 \%$ perderam o peso que esperavam e $19,5 \%$ recuperaram peso ao longo do tempo para depois estabilizarem o IMC. Do total dos operados bariátricos que se dispôs a responder ao questionário, 8,7\% disseram que não fariam a operação novamente por motivos particulares anexados ao questionário. Quanto ao preconceito em relação ao operado, 34,8\% percebem este fator como verdadeiro e fundamentado na visão de que a opeação é uma "mutilação necessária devido à falta de "força de vontade" e derivada da fraqueza de caráter do obeso". A operação permanece com o significado de impotência e corte como herança da psicanálise e da cultura. As pessoas que se dispuseram a responder às perguntas enviadas através da internet dividiram-se ao meio quanto aos benefícios dos grupos de apoio: parte defendeu a necessidade de dividir experiências comuns e parte defendeu $\mathrm{o}$ direito à privacidade, singularidade $\mathrm{e}$ sigilo (Figura 2).

\section{DISCUSSÃO}

De acordo com pacientes que buscam tratamento cirúrgico para a obesidade a ansiedade e a depressão aparecem como as doenças de maior prevalência nas entrevistas pré-operatórias. Essas referências diagnósticas informais não preenchem todos os critérios exigidos pelos códigos e manuais descritivos das doenças mentais, porém, estudos com candidatos à cirurgia bariátrica, avaliados por meio de testes e inventários auxiliam na concretização e quantificação dos dados. São os mais utilizados: inventário de Beck; inventário de personalidade multifásico de Minnesota (MMPI); escala de obesidade relacionada à comorbidades (AORC) e saúde relacionada à qualidade de vida (HRQOL).

Pesquisa da Universidade da Califórnia seguiu o modelo formal para concluir que a depressão atinge maior número entre os candidatos à operação e que estes pacientes tendem a ter pior resultado em perda de peso ou recuperar peso ao longo do tempo,

\begin{tabular}{|c|c|c|c|}
\hline $\mathrm{n}$ & Quesito & Sim ou maior & Não ou menor \\
\hline 1 & $\begin{array}{l}\text { Conhecimento sobre quantos procedimentos } \\
\text { bariátricos foram feitos }\end{array}$ & $93.5 \%$ sabiam & $6.5 \%$ não sabiam \\
\hline 2 & Conhecimento sobre o método cirúrgico & $\begin{array}{l}56.5 \% \text { tinham conhecimento sobre o método ao } \\
\text { qual foram submetidos }\end{array}$ & $\begin{array}{l}\text { 43.5\% não entenderam que a derivação intestinal } \\
\text { faz parte da operação de Fobi-Capella }\end{array}$ \\
\hline 3 & Preparo pessoal para a operação & $89.13 \%$ se sentiam preparados para seremoperados & $\begin{array}{l}10.87 \% \text { não se sentiam preparados para serem } \\
\text { submetidos à operação }\end{array}$ \\
\hline 4 & Motivação para a operação & $\begin{array}{l}\text { 100\% foram operados por razões psíquico-sociais } \\
\text { independentes de doenças orgânicas associadas }\end{array}$ & $\begin{array}{l}30.4 \% \text { foram operados por motivo de saúde física } \\
\text { independente indicação psico-social. }\end{array}$ \\
\hline 5 & Tratamento psicológico/psiquiátrico anterior & $48.8 \%$ receberam ou recebiam tratamento & $51.2 \%$ sem tratamento psicológico e ou psiquiátrico \\
\hline 6 & Tratamento psicológico e ou psiquiátrico atual & $\begin{array}{l}19.6 \% \text { em tratamento e com preferência à } \\
\text { psicoterapia }\end{array}$ & $\begin{array}{l}80.4 \% \text { não se submetiam atratamento no pós- } \\
\text { operatório }\end{array}$ \\
\hline 7 & $\begin{array}{l}\text { Conhecimento prévio de diagnóstico psicológico/ } \\
\text { psiquiátrico }\end{array}$ & $69.6 \%$ conheciam o diagnóstico & $\begin{array}{l}30.4 \% \text { foram diagnosticados na entrevista } \\
\text { psicológica }\end{array}$ \\
\hline 8 & Alteração no plano emocional após a operação & 71.7\% tiveram algum tipo de alteração emocional & $28.3 \%$ continuaram a mesma pessoa \\
\hline 9 & Dia do arrependimento & $26.08 \%$ referiram & 73.9 não referiram \\
\hline 10 & Transferência de compulsão/adição & $36.96 \%$ mudaram o foco da compulsão & 63.04\% não identificaram mudança de foco \\
\hline 11 & Pior fase & Dieta líquida 39\%Retorno anestésico 32.6\% & Restantes não referiram \\
\hline 12 & Aderência às recomendações & $50 \%$ adesão total & $50 \%$ adesão parcial ou ausente \\
\hline 13 & Problema alimentar pós-operatório & $28.3 \%$ algum tipo & $71.74 \%$ nenhum tipo \\
\hline 14 & Preparo psicológico em uma sessão & $32.6 \%$ ficaram satisfeitos com uma única consulta & $67.4 \%$ beneficiariam de mais preparo psicológico \\
\hline 15 & Divórcio & $\begin{array}{l}17.4 \% \text { se divorciaram (relatos de motivos não } \\
\text { vinculados) }\end{array}$ & $82.6 \%$ não tiveram rompimento de relacionamentos \\
\hline 16 & Preparo do parceiro & $67.4 \%$ preparados & $32.6 \%$ despreparados \\
\hline 17 & Preparo da família & $80.4 \%$ relataram familiares preparados & $19.6 \%$ com familiares despreparados \\
\hline 18 & Necessidade de agradar os demais & 43.5\% não têm mais necessidade de agradar & $56.5 \%$ persistem com esta necessidade \\
\hline 19 & Melhora do desejo sexual & 71.7\% melhoraram & $28.3 \%$ permanecerem iguais \\
\hline 20 & Satisfação com a perda de peso obtida & 93.5\% satisfeitos com a perda de peso & $6.5 \%$ insatisfeitos com a perda de peso \\
\hline 21 & Mudança de estilo de vida & $32.7 \%$ mudaram o estilo de vida & $67.3 \%$ não mudaram o estilo de vida \\
\hline 22 & Críticas por executar exercícios em exagero & $0 \%$ recebeu criticas & $100 \%$ não foram criticados \\
\hline \multirow[t]{2}{*}{23} & Recuperação de peso & & \\
\hline & $19.6 \%$ relataram recuperação de algum peso & $80.4 \%$ não recuperaram peso após a operação & \\
\hline 24 & Faria a operação de novo & $91.3 \%$ fariam & 8.7\% não fariam novamente \\
\hline 25 & Preconceito com o operado & $34.8 \%$ percebem preconceito & $65.2 \%$ não percebem preconceito \\
\hline 26 & Sente necessidade de grupos de apoio & $50 \%$ sentem & $50 \%$ não sentem \\
\hline
\end{tabular}

FIGURA 2 - Relação dos resultados obtidos com os questionários em 46 pacientes 
anulando parte ou todo sucesso cirúrgico. Também foi possível através do mesmo estudo perceber que a presença de depressão acabou por requerer tratamento deste transtorno do humor ou de quadros clínicos a ele associados ${ }^{3}$. Com os resultados obtidos foi possível inferir que o quadro de humor apresentado não era apenas resultado da condição clínica resultante da obesidade ou do efeito desta sobre a qualidade de vida, uma vez que os maiores índices de massa corporal não estavam correlacionados com a presença de depressão. A depressão deveria ser fisiologicamente ou metabolicamente associada às co-morbidades da obesidade sem uma relação direta causal.

No trabalho clínico são delineados dois grupos, um cujo fator de stress funciona como gatilho da obesidade e outro em que a obesidade funciona como fator de início da depressão com ou sem ansiedade. A operação pode constituir para os depressivos e/ ou ansiosos mais um evento existencial ou ritual de passagem que provoca euforia ou deprime. A psique é dinâmica em seu funcionamento e mesmo que provável em sua trajetória, nunca é previsível. Em entrevistas avaliativas é possível constatar no discurso dos pacientes que pessoas que tendem à depressão e distimia têm maior probabilidade de interpretar a operação como incompetência frente às dietas e mutilação de um órgão saudável. Diante deste significado serão passíveis de punição através de maus resultados e complicações operatórias por estarem "mexendo na obra divina".

Segundo estudo de Ronna Saunders ${ }^{9}$, do Centro de Mudança do Comportamento de Virginia, em período aproximado de seis meses após a operação, o padrão alimentar preponderante é o "beliscador". Este comportamento tende a substituir a compulsão a comer e predispõe à recuperação de peso. $O$ aumento do peso acontece sem consciência plena do operado que volta aos padrões de pensamento e comportamento antigos. A comida readquire os significados dissociados, ou boa ou ruim, e suscita culpa e/ou ansiedade. $\mathrm{O}$ ato de beliscar porções pequenas, que não promovem sensação de empanzinamento como ocorre na compulsão, inibe a percepção do limite alimentar. Estudo de Elkins et al. ${ }^{4}$ sobre aderência às recomendações pós-operatórias $44 \%$ dos operados apresentaram o hábito de "beliscar"; $40 \%$ não se exercitaram; $21 \%$ não foram ao grupo de suporte e $7 \%$ não tomaram vitaminas nos seis primeiros meses. $O$ mesmo grupo ao longo do primeiro ano permaneceu beliscando na porcentagem de 37\%; praticou exercício em $41 \% ; 25 \%$ passaram a frequentar o grupo de suporte e a ingestão de vitaminas subiu para $11 \%$.

No estudo do Departamento da Universidade da Pensilvânia coordenado por Thomas A. Wadden ${ }^{10}$ o comportamento de vomitar e purgar em operados bariátricos apareceu em um a dois terços. O vômito, tanto como reflexo quanto auto-induzido, não é considerado comportamento purgativo no paciente bariátrico, como o é na bulimia nervosa. Ele ocorre em função da comida que fica alojada no trato digestivo alto. Comer demais, não mastigar suficientemente ou comer determinados tipos de alimento, estão na base desse tipo de "devolução" alimentar. "Comer e cuspir" seria um comportamento prévio para evitar entalar-se com o alimento, ou mesmo não ter de vomitá-lo, como ocorre com a carne vermelha. No grupo brasileiro estudado pela autora foi possível constatar que a velocidade alimentar predispõe ao ato de vomitar, sendo a personalidade de base e o comportamento do operado, fundamentais para a determinação dos episódios de "devolução da comida". É possível inferir que alguns pacientes beneficiam-se desse efeito cirúrgico transformando-o em purgação.

Em se considerando o comer como adição e levando-se em conta que a nova proposta medicamentosa se compõe de naltrexona e bupropiona - a primeira bloqueadora de efeitos opióides utilizada no tratamento do alcoolismo e a segunda para depressão com especial efeito sobre tabagismo com estrutura molecular semelhante à anfetamina e dietilpropiiona ${ }^{8}$ -, há tendência de se considerar que o adicto à comida sofre de deficiência de dopamina. Alguns autores consideram, no entanto, que não se pode estabelecer cientificamente que uma pessoa possa ser "viciada em comida", mas os pacientes descrevem sua fissura por comida do mesmo modo que outros indivíduos relatam a necessidade biológica da droga. Talvez seja esse o caminho da compreensão da vulnerabilidade às drogas. Hoje é de conhecimento da ciência que quanto mais jovem é um indivíduo, mais intenso se torna o mecanismo de suporte ao stress através da comida ${ }^{1}$. A utilização da bupropiona associada ao topiramato em protocolo de balão intragástrico tem auxiliado pacientes nos períodos finais da estadia do recurso, bem como na manutenção da perda de peso.

O uso da comida alivia o desconforto provocado pela disforia, mas como as demais drogas, ao longo do consumo provocam disfunções cognitivas. Os transtornos alimentares, que têm claro vínculo com depressão, ansiedade e estados mistos do humor, também levam às mesmas condições 5 .

Assim como em qualquer doença crônica em que a aderência ao tratamento e o seguimento são difíceis e constituem foco do tratamento, em qualquer comportamento aditivo a prevenção de recaídas é o cerne do programa terapêutico. Para que as recaídas não venham a ocorrer Alexander ${ }^{2}$ em seu "Guia Prático para a Vida Após a Cirurgia Bariátrica" é assertiva: "... Você nunca mais comerá como os outros novamente. Você é especial e precisa de considerações especiais. Faça as pazes com isso e você se sentirá menos ansioso. Tente olhar para suas diferenças individuais de modo positivo. Mantenha os olhos na balança e tenha um diálogo positivo consigo mesmo..."

A cirurgia bariátrica mudou seu panorama inicial. Sua clientela vem sendo de IMC menor, mais 
esclarecida, mais temerosa em relação às consequências da obesidade e menos receosa em relação à operação. O discurso do cliente bariátrico é a saúde, mas é na aceitação social e na dinâmica psicológica que está o centro dessa busca da troca de corpo. A cirurgia bariátrica é um braço da busca pela juventude.

A sexualidade recebe pontuação positiva na maioria das investigações após a cirurgia bariátrica, mas casos em que há sobra excessiva de pele e falta de condições financeiras para operações consideradas estéticas, levam ao retraimento da pessoa e até ao sentimento de mais uma falência. Há consenso quanto ao aspecto maior agilidade, maior facilidade para execução do ato sexual e maior capacidade de fornecer e receber prazer, mas esses critérios dizem respeito ao plano físico ligado ao sexo. A reavaliação do parceiro sexual, o bem-estar durante o ato, a auto-estima e espontaneidade durante o intercurso sexual são pouco explorados pela literatura e parecem ser influenciados pela imagem corporal e pelo relacionamento entre os pares. A queda de desejo sexual em algumas mulheres pode estar relacionada ao emagrecimento rápido que provoca declínio abrupto dos níveis de estrogênio que é necessário para a produção de testosterona. A ligação entre testosterona e desejo sexual feminino é inquestionável e sua alteração fica garantida mediante a queda dos níveis de estrogênio promovida pela rápida perda de peso ${ }^{6}$. Mas o desejo sexual ainda é algo muito complexo e não pode ser reduzido ao tamanho do corpo e alguns poucos hormônios, haja vista que magras também tem déficit de desejo, problemas na manutenção da libido e dificuldades com o orgasmo. Um terço dos homens parece corrigir disfunções metabólicas relacionadas à obesidade após a operação, sendo a perda de peso a causa da correção. A imagem corporal parece não interferir do mesmo modo sobre a espontaneidade durante o intercurso sexual para os homens como o faz para as mulheres ${ }^{7}$.

No que tange aos relacionamentos afetivos, ainda a média de casais que melhoram sua relação é maior que as separações conjugais. São estudos empíricos que mostram também a capacidade que a mudança em um dos pares tem de propiciar a quebra da relação, mesmo que esta mudança seja considerada boa ${ }^{10}$.

\section{CONCLUSÕES}

Pode-se inferir que as motivações que levam os indivíduos a procurar a cirurgia bariátrica, são em sua maioria, calcadas nos retornos sociais, nos padrões estéticos e no desejo psicológico de mudança de vida. Uma vez concretizada a mudança de vida, a motivação se acaba, mas não garante a satisfação existencial, busca pertinente aos seres humanos. Pacientes bariátricos podem apresentar maiores distorções perceptivas corporais que fazem com que não notem sua recuperação de peso, ou se defendam dessa percepção, de modo a só buscar auxílio quando o ganho ponderal é excessivo e perceptivo para o social. Com recuperação de peso tendem a buscar outros recursos cirúrgicos ou clínicos a aceitar que seu consumo alimentar possa estar calcado em funcionamentos cerebrais e emocionais, como adição à comida.Também é possível inferir que o preparo para o atendimento de cônjuges e familiares reclama um espaço na formação de profissionais da área do tratamento cirúrgico da obesidade, para que a prevenção às recaídas possa ser foco terapêutico. $O$ tempo de preparo pré-operatório se estende para além de uma única consulta psicológica e nutricional, mas aumenta sua eficácia.

\section{REFERÊNCIAS}

1. Alexander C L, Transfer of Addiction (Transfer of Vice), Alcohol and Smoking. In: The Emotional First + Aid Kit. A Practical Guide to Life After Bariatric Surgery. Second Ed. Matrix Medical Communications, 2009.p.136

2. Alexander C L, Weight Maintenance.In: The Emotional First + Aid Kit. A Practical Guide to Life After Bariatric Surgery. Second Ed. Matrix Medical Communications, 2009.p.151

3. Ali MR, J. Rasmussen J J, Monash JB, Fuller WD .Depression is associated with increased severity of co-morbidities in bariatric surgical candidates. S O R D. 2009; 5:559-564

4. Elkins G, Whitfield P, Marcus J, Symmonds R, Rodrigues J, Cook T. Noncompliance with behavioral recommendations following bariatric surgery. Obes Surg. 2005; 15:546-551

5. Gold MS, Oppenheim HC, Frost-Pineda K. Obesity and Substance Use Disorders. In: McElroy, SL, Allison DB, Bray GA. Obesity and Mental Disorders. Taylor \& Francis Group, New York/London. 2006.p.103-122.

6. Meana M, Ricciardi L. Changing The Dynamics in Your Inner Circle. Your Change Becomes Everybody Else's In: Meana M, Ricciardi L. Obesity Surgery. Stories of Altered Lives. University of Nevada Press. 2008.p.88-130

7. Meana M, Ricciardi L. Deconstructing the Self. Who Was I and What Am I Becoming?. In: Meana M, Ricciardi L. Obesity Surgery. Stories of Altered Lives. University of Nevada Press. 2008. p. 131158

8. Sadock B, Sadock V, Sussman N. Bupropiona In: Manual de Farmacologia Psiquiátrica de Kaplan \& Sadock. Sadock B, Sadock V, Sussman N. Artmed. Porto Alegre. 2007.10: 131-140

9. Saunders R. "Grazing" A High-Risk Behavior. Allied Health. Obes Surg.2003.14:98-102

10. Wadden TA, Sarwer DB , Fabricattore NA, Jones L, Stack R, Williams NS. Psychosocial Behavioral Status and of Patients Undergoing Bariatric Surgery: What to Expect Before and After Surgery. The Med Clin of N.A. 2007.91: 451-4 